\title{
Modality effects in the coding and reproduction of rhythms
}

\author{
ARTHUR M. GLENBERG, STUART MANN, LISA ALTMAN, \\ TIM FORMAN, and SEAN PROCISE \\ University of Wisconsin, Madison, Wisconsin
}

\begin{abstract}
The temporal coding assumption is that time of presentation is coded more accurately for auditory events than for visual events. This assumption has been used to explain the modality effect, in which recall of recent auditory events is superior to recall of recent visual events. We tested the temporal coding assumption by examining the coding and reproduction of quintessentially temporal stimuli-rhythms. The rhythms were produced by sequences of short and long auditory stimuli or short and long visual stimuli; in either case, the task was to reproduce the temporal sequence. The results from four experiments demonstrated reproduction of auditory rhythms superior to that of visual rhythms. We conclude that speech-based explanations of modality effects cannot accommodate these findings, whereas the findings are consistent with explanations based on the temporal coding assumption.
\end{abstract}

In classic information processing models of memory (e.g., Atkinson \& Shiffrin, 1968), the input modality of information plays little role after the information is coded in short-term store. In fact, early work on the modality effect (e.g., Crowder \& Morton, 1969) seemed to confirm this limited role: Auditory information is recalled better than is visual information, but only at the end of a list of items, supposedly indicating recall from a sensory memory or a short-term memory. In the intervening years, we have found, however, that modality plays a role throughout the cognitive system. For example, J. M. Gardiner and Gregg (1979) have demonstrated longlasting modality effects that are robust in the face of interference. Glenberg and Fernandez (1988) reported modality effects in position judgments and in frequency estimations. In this article, we report a new type of modality effect, one that offers support for a general interpretation of modality effects in memory. The new effect is that the coding and reproduction of rhythmic stimuli are more accurate for auditory rhythms than for visual rhythms. This supports the interpretation that modality effects reflect the fact that coding of temporal information for auditory events is superior to that for visual events.

Glenberg and Swanson (1986) proposed an account of modality effects based on what we will call the temporal coding assumption: Coding of time of presentation is more accurate for auditory events than for visual events. Evidence for this claim can be found in a variety of paradigms using a variety of manipulations. For example, Metcalfe,

This research was funded by National Science Foundation Grant BNS 8416300 and University of Wisconsin Graduate School Grant 890200 awarded to Arthur Glenberg. We thank Michael Davis, Lisa Knudson, Nancy Lightfoot, and Rebecca Mauer for their help in designing these experiments. Requests for reprints may be sent to Arthur Glenberg, Department of Psychology, University of Wisconsin, Madison, WI 53706 .
Glavanov, and Murdock (1981) presented stimuli in different spatial locations. Auditory stimuli were recalled better than were visual stimuli when recall was by temporal position, but this difference was eliminated when recall was by spatial location. Glenberg and Swanson (1986) manipulated the duration of the intervals separating successive to-be-remembered items on a list. This manipulation greatly affected recall of auditory items (enhancing the recency effect), but had little effect on the recall of visual items. Glenberg and Fernandez (1988) discovered modality effects in an order judgment task. After a list of stimuli was presented, subjects judged if the items in a re-presented pair were in the correct order. They found an auditory superiority both for the stimuli presented at the end of the list and for those presented at the beginning of the list. Furthermore, the auditory superiority increased with duration of the interitem intervals. Thus, across these paradigms, the temporal coding assumption is supported by the fact that there was greater sensitivity to temporal manipulations of auditory events than there was to temporal manipulations of visual events (but see Crowder and Greene, 1987, for a dissenting opinion).

These demonstrations of effects consistent with the temporal coding assumption rely on relatively long-term memory. Even in the immediate recall of a short list of items, the modality effect is enhanced by serial recall, which delays recall of the end-of-list items where the largest modality effect is found. The temporal coding assumption leads us to expect, however, effects much closer to the perception of the events. That is, it proposes that time of occurrence is coded (not just remembered) more accurately for auditory than for visual stimuli. This expectation led us to look for modality effects in a domain that is quintessentially temporal-the perception and reproduction of rhythms. 
A thythm is produced by a sequence of stimuli that can be identical except for their temporal durations. Thus, perceiving a rhythm requires coding of temporal information and not much more. Our expectation is that rhythms produced by a sequence of long- and short-duration auditory stimuli will be easier to perceive and reproduce than will identical rhythms produced by a sequence of longand short-duration visual stimuli. ' In the General Discussion section, we present the implications of our findings for explanations of the modality effect.

In our rhythm task, subjects were presented with a sequence of $n(n=5-8)$ stimuli. Each stimulus was presented for either a long duration $(750 \mathrm{msec})$ or a short duration ( $250 \mathrm{msec})$, and each was followed by an empty, short $(250 \mathrm{msec})$ interstimulus interval. For an auditory sequence, the stimuli were long- and short-duration tones. For a visual sequence, the stimuli were asterisks that were presented on a display terminal for a long or short duration. Immediately following the last stimulus, subjects reproduced the rhythm, using button presses to indicate the sequence of long- and short-duration stimuli.

This task is not ideal for studying the perception and coding of time of presentation; there is still a memory component. The memory component is minimized, however, in that there are only two stimuli (long and short), and the retention interval is very short.

In overview, we conducted four experiments. Experiments 1 and 2 demonstrated the basic finding that performance is better for auditory rhythms than for visual rhythms. In addition, these experiments ruled out explanations of the effect based on modality differences in the detectability of onsets and offsets of auditory and visual stimuli. In Experiment 3, we explored the possibility that the effect is due to response requirements and that it may reflect differential experience in coding auditory and visual rhythms. Experiment 4 demonstrated a correspondence between the rhythm modality effect and the modality effect found in recall tasks.

\section{EXPERIMENT 1}

Experiment 1 was designed to determine if auditory superiority in the rhythm task is due to perceived change from background. Our experience in the task indicated that the auditory stimulus is more alerting than is the visual stimulus (cf. Posner, Nissen, \& Klein, 1976), perhaps because the auditory stimulus is a greater change from its background (silence) than the visual stimulus is from its background (ambient visual stimulation). This alerting could enhance auditory performance in at least two ways. First, the alerting may increase the accuracy of the perception of duration, because the actual onset of the auditory stimulus is noted more accurately than is the actual onset of the visual stimulus. Second, the alerting may recruit attentional resources to enhance the coding and memory of auditory duration information (whether or not the duration is perceived more accurately than is the duration of the visual stimulus).

We tested these ideas by manipulating the background. In the auditory-noise-background condition, the stimuli (both auditory and visual sequences) were presented while white noise was being played. The white noise should decrease the change from background for the auditory stimulus and thereby reduce performance on the auditory sequences. In the visual-noise-background condition, the stimuli were presented while visual noise (flickering random dot patterns) appeared on the display screen. This background should selectively reduce performance of the visual stimuli. Finally, in the no-background condition, the stimuli were presented in a quiet, dark environment. Our reasoning did not depend on equating the auditory and visual backgrounds or changes in the backgrounds. Instead, we intended to determine if changing the background in modality selectively affects coding and reproduction of rhythms presented in that modality.

\section{Method}

Subjects. The subjects were 20 students enrolled in introductory psychology courses at the University of Wisconsin-Madison. They received credit toward a research requirement for participating in the experiment.

Materials. The subjects were seated approximately $55 \mathrm{~cm}$ in front of a visual display terminal and two loudspeakers. All subjects wore a limited-vision mask that restricted vision to a $6 \times 12 \mathrm{~cm}$ area of the display screen. This mask was used to eliminate ambient visual stimulation in the no-background condition, but all subjects wore the mask throughout the experiment.

The auditory stimulus was a tone of complex composition. ${ }^{2}$ The intensity of the tone at the ear was approximately $77 \mathrm{~dB}(\mathrm{~A})$. The tone was presented for either $250 \mathrm{msec}$ (short) or $750 \mathrm{msec}$ (long). During the block of trials corresponding to the auditory-noisebackground condition, white noise was played continuously at an intensity of approximately $75 \mathrm{~dB}(\mathrm{~A})$.

The visual stimulus was a single asterisk (produced using an Apple II's Turtlegraphics font) centered in the visible area of the display screen. In the visual-noise-background condition, a flickering random dot pattern appeared on the display screen, except for a $.5 \times 1.2 \mathrm{~cm}$ rectangle in which the asterisk appeared. The random dot pattern appeared after the ready signal (see below) and was presented for $500 \mathrm{msec}$ before the first stimulus and throughout the presentation of the stimuli.

Each sequence began with a ready signal consisting of a warning tone, a visual message indicating whether the following sequence would consist of tones or asterisks, and the number of tones or asterisks (five, six, or seven). The visual message was displayed for $500 \mathrm{msec}$. The ready signal was followed by a 500 -msec delay that was filled with the visual noise background in that condition.

Each sequence consisted of five, six, or seven stimuli. The sequence always contained at least one long and one short stimulus. Other than this constraint, the sequences were generated randomly for each subject. Following the last stimulus in a sequence there was a 500-msec delay before the subject was prompted to respond (the delay was imposed to eliminate masking due to the prompt). The prompt consisted of a series of five, six, or seven dashes on the screen. For each serial position in the sequence, the subjects responded with a left-hand button to indicate a short stimulus and a right-hand button to indicate a long stimulus. On responding, the dash in the corresponding serial position was replaced by an $S$ or 
an L. The subject responded in serial order, but, by pressing both buttons simultaneously, he/she could erase the sequence and start over. When satisfied with the response, the subject pressed a third button to record the response and initiate the ready signal for the next trial.

Design. Except for the use of two experimenters, all factors were manipulated within subjects. The order of the three background conditions (auditory noise, visual noise, no noise) was counterbalanced across subjects. Within a background condition, the modality of the sequences (auditory or visual) was blocked and counterbalanced. Within each modality, there were eight sequences of each length $(5,6$, or 7$)$. These sequences were blocked by length and were always presented in order starting with Sequence Length 5.

Before each change of background, the subjects were given a brief break and were informed of the nature of the upcoming condition. In addition, before the experimental trials began, the subjects were given practice (in the no-noise condition) on two sequences of tones and two sequences of asterisks. At the end of the experiment, they were asked if they had closed their eyes to avoid the visual noise background during the auditory trials. No subject admitted to doing this.

\section{Results}

The first two sequences of each length for each modality for each background condition were treated as practice and were not scored. The responses for the remaining six sequences were scored in two ways. First, a sequence was scored as 1 if the responses (short or long) in all serial positions were correct (or 0 otherwise). We will refer to this as the completely correct score. Second, we scored the response in each serial position as correct or not, so that we could examine serial position curves. In the analyses of the completely correct variable, experimenter was treated as a factor (with the reduction in degrees of freedom for various error terms). Neither the main effect nor any of its interactions were significant. This factor was not included in analyses by serial position. All statistical analyses were conducted with the probability of a Type I error set equal to .05 , and the dependent variable was always number correct (rather than proportions as indicated in the figures).

The data from the completely correct measure are illustrated in Figure 1. Note that there was a general advantage for the auditory sequences $(.70)$ over the visual sequences $(.58)\left[F(1,18)=18.94, M S_{\mathrm{e}}=2.63\right]$. This result demonstrates auditory superiority in the coding and reproduction of rhythmic stimuli. There also was a highly significant effect of sequence length $[F(2,36)=51.37$, $\left.M S_{\mathrm{e}}=1.32\right]$.

The change-from-background hypothesis predicts a decrement in performance when the stimulus modality matches the background modality. In other words, stimulus modality and background condition should interact. Although none of the two-factor interactions were significant ( $p s>$.19), the three-factor, background condition $\times$ modality $\times$ length interaction was significant $[F(4,72)=$ $\left.2.98, M S_{\mathrm{e}}=1.21\right]$. Nonetheless, this interaction does not offer much support to the change-from-background hypothesis. Note that, except at Sequence Length 7, performance on the auditory sequences in the auditory back-

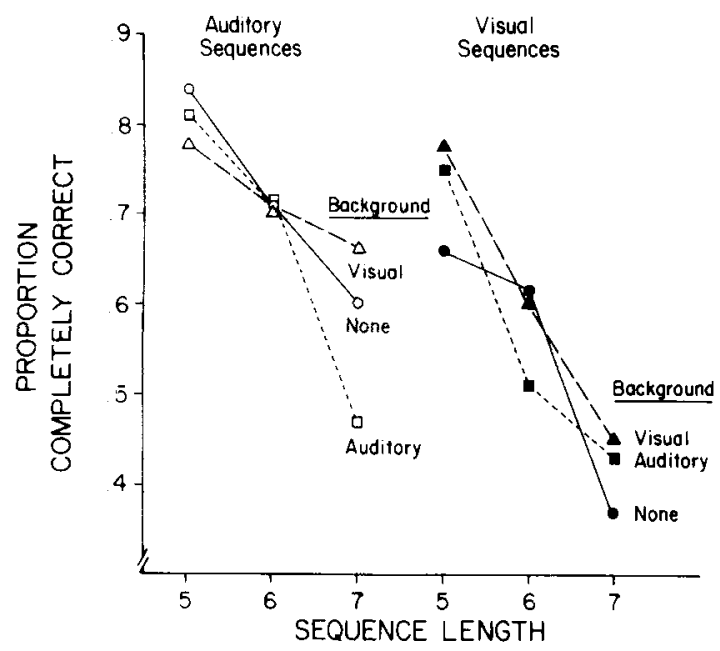

Figure 1. Data from Experiment 1: Proportions of sequences recalled exactly as presented as a function of sequence length, modality of presentation, and background condition.

ground condition was within a few percentage points of performance in the visual-noise-background and no-noisebackground conditions. Considering performance on the visual sequences, performance in the visual-noisebackground condition (.61) was slightly larger than it was in the auditory-noise-background condition (.56) and the no-noise-background condition (.55).

The results from the serial position analysis, averaged over sequence length, are illustrated in Figure 2. For each sequence length, we scored the first two and last three serial positions so that we could include sequence length as a factor in the analysis of variance. There was a main effect for sequence modality, indicating auditory superiority $\left[F(1,19)=22.23, M S_{\mathrm{e}}=1.09\right]$; a main effect for length, indicating a decrease in performance with the longer lengths $\left[F(2,38)=36.51, M S_{\mathrm{e}}=1.10\right]$; and a main effect of serial position, indicating a decrease in recall across the positions $\left[F(4,76)=34.77, M S_{c}=1.31\right]$.

A number of interactions were significant. First, the modality $\times$ serial position interaction was significant, indicating an increase in the auditory advantage toward the end of the sequence $\left[F(4,76)=4.34, M S_{\mathrm{e}}=.64\right]$. There was also a significant sequence length $\times$ serial position interaction $\left[F(8,152)=5.56, M S_{\mathrm{e}}=.70\right]$. This interaction was produced by the fact that the three lengths did not differ much in the initial serial position $(.97, .95$, and .95 , for Lengths 5,6 , and 7 , respectively), but did differ in the final serial position $(.87, .81$, and .77$)$. Finally, the three-factor background condition $\times$ sequence modality $\times$ length interaction was sigrificant $[F(4,76)$ $=2.87, M S_{\mathrm{e}}=.88 \mathrm{~J}$. As with the corresponding interaction in the completely correct analysis, this interaction was not systematic: In the no-noise background, the auditory superiority was greatest for sequences of Length 5 , in the auditory background condition the auditory superiority was greatest for sequences of Length 6 , and in the visual background condition, the auditory superiority was 


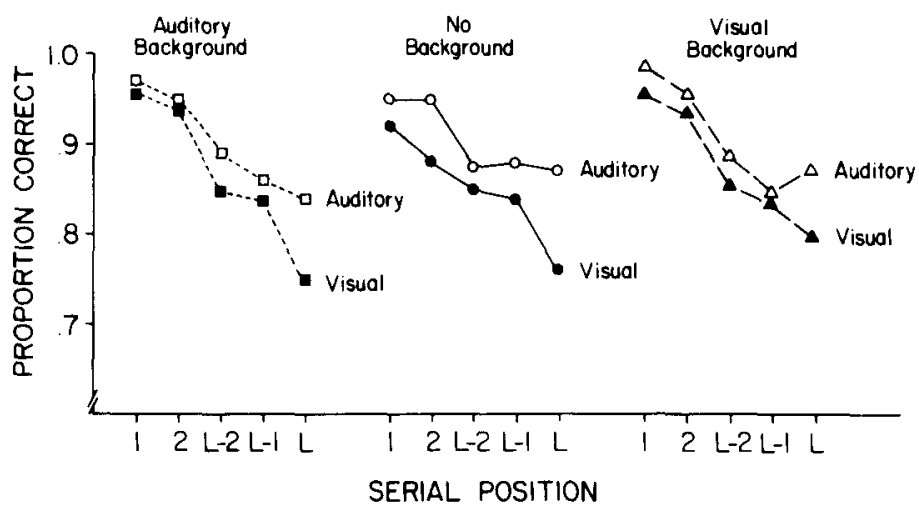

Figure 2. Data from Experiment 1: Proportion of stimuli correct at each input serial position as a function of modality of presentation.

greatest for sequences of Length 7. Table 1 provides the means contributing to these interactions.

The three-factor interaction notwithstanding, there is little support for the change-from-background hypothesis. Across the three background conditions, average performance on the auditory sequences ranged only from .90 to .92 , and average performance on the visual sequences ranged only from .86 to .87 . Nonetheless, the serial position curves are notably different from standard serial position curves in two respects. First, there is little or no recency effect. Second, the auditory superiority is found throughout the serial position curve, although as indicated by the significant modality $\times$ serial position interaction, auditory superiority does increase toward the end of the sequence. We will discuss this further after presentation of Experiment 2 and in the General Discussion section.

Table 1

Means from Experiment 1

\begin{tabular}{|c|c|c|c|c|c|c|}
\hline \multirow[b]{2}{*}{ Modality } & \multirow{2}{*}{$\begin{array}{l}\text { Sequence } \\
\text { Length }\end{array}$} & \multicolumn{5}{|c|}{ Serial Position } \\
\hline & & 1 & 2 & Last 2 & Last 1 & Last \\
\hline \multicolumn{7}{|c|}{ No Background } \\
\hline Auditory & $\begin{array}{l}5 \\
6 \\
7\end{array}$ & $\begin{array}{l}.98 \\
.95 \\
.92\end{array}$ & $\begin{array}{l}.97 \\
.98 \\
.90\end{array}$ & $\begin{array}{l}.93 \\
.88 \\
.82\end{array}$ & $\begin{array}{l}.92 \\
.89 \\
.82\end{array}$ & $\begin{array}{l}.96 \\
.82 \\
.82\end{array}$ \\
\hline Visual & $\begin{array}{l}5 \\
6 \\
7\end{array}$ & $\begin{array}{l}.93 \\
.94 \\
.89\end{array}$ & $\begin{array}{l}.90 \\
.90 \\
.89\end{array}$ & $\begin{array}{l}.88 \\
.92 \\
.76\end{array}$ & $\begin{array}{l}.89 \\
.87 \\
.77\end{array}$ & $\begin{array}{l}.80 \\
.79 \\
.68\end{array}$ \\
\hline \multicolumn{7}{|c|}{ Auditory Background } \\
\hline Auditory & $\begin{array}{l}5 \\
6 \\
7\end{array}$ & $\begin{array}{l}.98 \\
.97 \\
.96\end{array}$ & $\begin{array}{l}.96 \\
.93 \\
.95\end{array}$ & $\begin{array}{l}.94 \\
.91 \\
.81\end{array}$ & $\begin{array}{l}.92 \\
.90 \\
.77\end{array}$ & $\begin{array}{l}.91 \\
.83 \\
.77\end{array}$ \\
\hline Visual & $\begin{array}{l}5 \\
6 \\
7\end{array}$ & $\begin{array}{l}.98 \\
.94 \\
.96\end{array}$ & $\begin{array}{l}.98 \\
.89 \\
.93\end{array}$ & $\begin{array}{l}.94 \\
.86 \\
.76\end{array}$ & $\begin{array}{l}.97 \\
.78 \\
.79\end{array}$ & $\begin{array}{l}.83 \\
.73 \\
.69\end{array}$ \\
\hline \multicolumn{7}{|c|}{ Visual Background } \\
\hline Auditory & $\begin{array}{l}5 \\
6 \\
7\end{array}$ & $\begin{array}{l}.98 \\
.98 \\
.99\end{array}$ & $\begin{array}{l}.97 \\
.94 \\
.98\end{array}$ & $\begin{array}{l}.93 \\
.92 \\
.83\end{array}$ & $\begin{array}{l}.91 \\
.85 \\
.81\end{array}$ & $\begin{array}{l}.87 \\
.87 \\
.90\end{array}$ \\
\hline Visual & $\begin{array}{l}5 \\
6 \\
7\end{array}$ & $\begin{array}{l}.98 \\
.93 \\
.96\end{array}$ & $\begin{array}{l}.94 \\
.97 \\
.92\end{array}$ & $\begin{array}{l}.96 \\
.86 \\
.77\end{array}$ & $\begin{array}{l}.92 \\
.83 \\
.77\end{array}$ & $\begin{array}{l}.86 \\
.82 \\
.72\end{array}$ \\
\hline
\end{tabular}

\section{EXPERIMENT 2}

Like Experiment 1, Experiment 2 tested the changefrom-background hypothesis. In this experiment, however, the manipulation was of stimulus energy, rather than of type of background.

The auditory stimulus was either loud or soft. The change-from-background hypothesis predicts better performance with the loud stimulus. The visual stimulus was either a single asterisk or many asterisks on the display screen. The hypothesis predicts better performance in the many-asterisks condition.

\section{Method}

Subjects. The subjects were 22 students obtained from the same source as were those in Experiment 1.

Materials. In the soft condition, the auditory stimuli were identical to those used in the no-noise-background condition of Experiment 1 , except that the intensity of a stimulus tone was approximately $55 \mathrm{~dB}(\mathrm{~A})$. In the loud condition, the intensity was set to approximate $85 \mathrm{~dB}(\mathrm{~A})$. The visual stimulus (asterisk) in the single condition was exactly the same as the visual stimulus in the nonoise condition of Experiment 1 . In the many condition, the visual stimulus consisted of an array of asterisks that completely filled the visible part of the display screen.

Design. Except for assignment to one of the two experimenters, all factors were manipulated within subjects. The two auditory conditions (loud and soft) and the two visual conditions (single and many) were blocked, but the order of the blocks was counterbalanced across subjects. In addition, we counterbalanced the order of the two conditions within a modality block. Within a condition, the subjects were presented with 10 sequences of each of the lengths $(5,6$, and 7 , in that order). The structure of each sequence (ready signal, sequence, response interval) was identical to that used in Experiment 1.

\section{Results}

The results of the completely correct scoring are illustrated in Figure 3. The only significant effects were for sequence length $\left[F(2,40)=33.06, M S_{\mathrm{e}}=2.67\right]$ and condition $\left[F(3,60)=4.27, M S_{\mathrm{e}}=3.21\right]$. We further examined the main effect of condition using planned orthogonal contrasts. The difference between the auditory and the visual sequences was significant $[F(1,60)=12.05]$, 


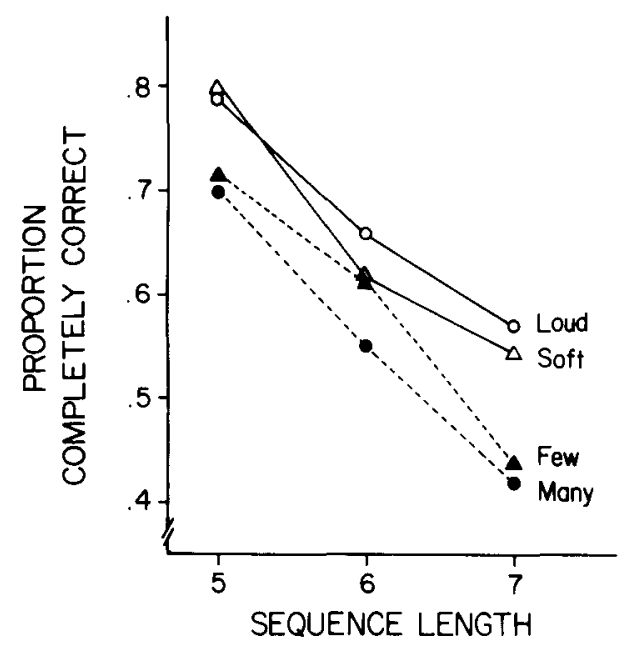

Figure 3. Data from Experiment 2: Proportions of sequences recalled exactly as presented as a function of sequence length and modality of presentation.

but the difference between the loud and soft conditions was not significant, nor was the difference between the single and many conditions (both $F \mathrm{~s}<1$ ). Thus, once again, there is no support for the change from background hypothesis.

The results of the serial position analysis (first two and last three positions) are illustrated in Figure 4 . There were significant main effects for condition $[F(3,60)=8.11$, $\left.M S_{\mathrm{e}}=2.67\right]$, length $\left[F(2,40)=22.11, M S_{\mathrm{e}}=2.14\right]$, and position $\left[F(4,80)=17.59, M S_{\mathrm{e}}=1.93\right]$. Although it appears that the auditory advantage increased near the end of the sequence, the condition $\times$ position interaction was not significant $\left[F(12,240)=1.62, M S_{\mathrm{e}}=.72\right.$, $p=.09]$. As in Experiment 1, there was little recency effect, and the auditory advantage was found at most serial positions.

\section{Discussion of Experiments 1 and 2}

The most important result was that there was an auditory superiority in the coding and reproduction of rhythms. The result was somewhat smaller than we had expected

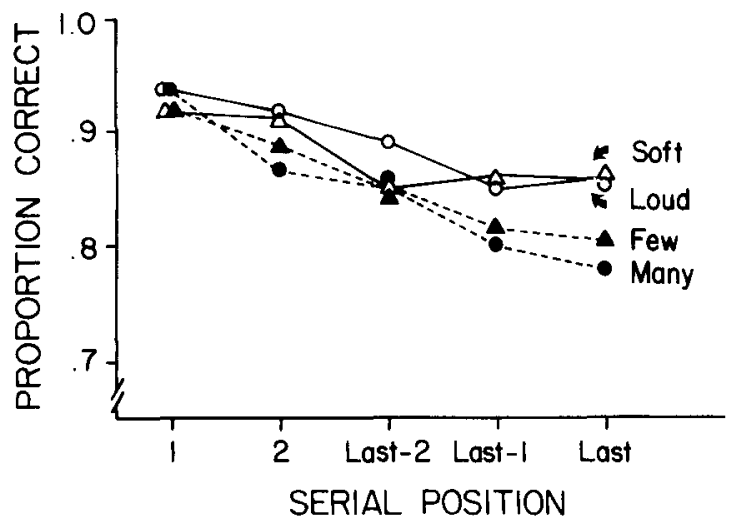

Figure 4. Data from Experiment 2: Proportion of stimuli correct at each input serial position as a function of modality of presentation. given our own experience in the task. Our subjective impression is that there is no need to engage an active, effortful coding process for the auditory stimuli-the rhythm simply impresses itself upon one. In contrast, the visual stimuli seem to require attention and effortful processing to code the durations as long or short. When queried, all of our subjects felt similarly. Although small in absolute terms, the auditory superiority was very consistent.

The auditory superiority is remarkable in that the physical information that must be coded is exacty the same for the auditory and visual sequences: In either case, the subject is responsible only for the duration of the events. This should be contrasted with standard modality effects in which the physical stimuli (e.g., a spoken word and a written word) are markedly different for the two modalities. Consistent with the temporal coding assumption, the auditory modality apparently is more specialized for coding temporal information than is the visual modality.

The results of Experiments 1 and 2 offer little support for the change-from-background hypothesis. Although the auditory stimuli may be more alerting, it is not because there is a greater change from the auditory background than there is from the visual background.

\section{EXPERIMENT 3}

The auditory superiority may not be due to any intrinsic difference between coding of auditory and visual stimuli (as implied by the temporal coding assumption). Instead, it may simply reflect differential practice. Most of us have had extensive practice identifying auditory thythms from listening to music, poetry, or a well-tuned engine. Rhythms carried by the onsets and offsets of visual events (e.g., a flickering television screen) seem rarer. Experiment 3 was designed to test this notion by giving the subjects extensive practice in the rhythm task over 2 days.

We examined the data for two indicants of differential amounts of previous learning to code rhythms. First, if it is just a matter of performance in the visual condition catching up to performance in the auditory condition, we should observe equal asymptotes after practice. Suppose, however, that performance does not reach asymptotic levels after 2 days of practice. In this case, we are forced to consider whether performance in the visual condition is improving faster than performance in the auditory condition (indicating that visual performance is at an earlier part of a negatively accelerated learning curve). To do this, we used auditory sequences of Lengths 6,7 , and 8 , and visual sequences of Lengths 5,6 , and 7 . We made the auditory task more difficult so that we could find auditory and visual conditions that started at about the same performance level (e.g., Auditory Length 7 and Visual Length 6), enabling us to examine the learning curves for different rates of learning.

We also manipulated the type of response the subjects made after the sequence. In Experiments 1 and 2, the subjects responded by using two keys to indicate the sequence of long and short stimuli. Perhaps this dichotomous re- 
sponse encouraged unnecessary verbal recoding of visual stimuli into the words long and short, leading to poorer performance with the visual stimuli. In Experiment 3, half of the subjects used the dichotomous (short-long) response mode, and half used a continuous response mode. In the continuous response mode, the subject pressed one key for a duration that matched the remembered duration of the first stimulus. The key then was released and pressed again to indicate the duration of the second stimulus, and so on. The duration of each keypress was indicated on the display terminal by a line whose horizontal extent increased while the key was pressed. Lifting the key moved the cursor down the display screen where the next duration would be indicated. The various line lengths (indicating durations) were continuously displayed during the response interval (much like the Ss and Ls in the dichotomous response mode), and the subject could start over at any time. In addition, the instructions never mentioned the words long and short, and never referred to the fact that only two durations were used. Thus, we tried to eliminate any encouragement to verbally recode the stimuli.

\section{Method}

Subjects. The subjects were 24 students from the same source as for Experiments 1 and 2.

Materials. The stimuli were analogous to the loud-tones and many-asterisks conditions from Experiment 2. Auditory sequence lengths were 6,7 , and 8 , and the visual sequence lengths were 5 , 6 , and 7.

Design. The subjects were assigned randomly to one of two experimenters and to a response mode (dichotomous or continuous). For the subjects in the continuous response mode, the instructions were slightly modified to remove any reference to the number of different stimulus durations, and the words long and short were not used. The other factors were manipulated within subjects. One variable was day; the subjects participated in the experiment on 2 consecutive days. Each day, the subjects experienced three blocks of sequences. A block consisted of six auditory sequences at each length $(6,7$, and 8$)$ and six visual sequences at each length $(5,6$, and 7). Within a block, the order of the auditory and visual sequences was counterbalanced over subjects (for one particular subject, however, the order remained the same across all blocks and days), but the sequences were always blocked by modality and were always presented in the order of increasing sequence length. In addition, before these trials started, the subjects had practiced on two auditory trials and two visual trials. All other aspects of the design and procedure were identical to those of Experiment 2.

\section{Results}

The first sequence in every group of six was considered as practice and was not scored; otherwise, scoring for the subjects in the dichotomous response condition was identical to that used in Experiments 1 and 2. A different procedure was used for the subjects in the continuous response condition. The raw data consisted of the durations for which the response key was pressed. We needed to find a cutoff duration so that any longer duration key press would be classified as long and any shorter duration key press would be classified as short. To find a cutoff for each subject, we used an iterative procedure that maximized the proportion of matches between the actual stimulus (long or short) and the classification. After the responses were classified, they were scored exactly as in the dichotomous response condition.

The results from the completely correct scoring are displayed in Figure 5. First, note that learning has, apparently, reached an asymptote by the end of Day 2. In fact, many of the curves show a slight downturn between Blocks 2 and 3 on Day 2. Thus, we need not worry about having to compare the rates of learning; instead, we can concentrate on the asymptotic levels. Second, for sequences of comparable lengths, performance in the auditory conditions exceeds performance in the visual conditions at the asymptotes and in the initial parts of the learning curves. Thus, the auditory superiority is unlikely to reflect only differential extraexperimental practice. On the other hand, note that there is some evidence (particularly in the dichotomous response condition) that performance in the visual conditions showed an initial spurt from Block 1 to Block 2 (on Day 1) not matched by the auditory conditions. Finally, note that, in the continuous response mode, the auditory superiority was as large and as consistent as it was in the dichotomous response mode. We learn from this last result that the auditory superiority was not due to the fact that the subjects were forced to use a dichotomous and verbally codable response mode.

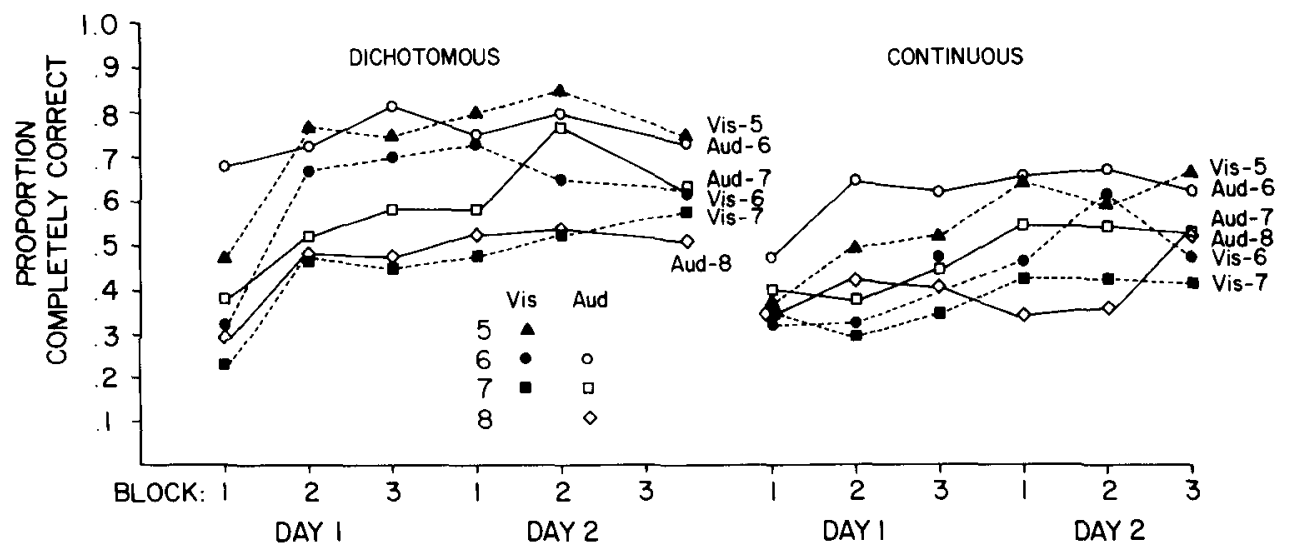

Figure 5. Data from Experiment 3: For each response mode, proportion of sequences recalled exactly as presented as a function of modality of presentation, sequence length, and practice. 
These observations are supported by analyses of variance conducted separately for the dichotomous and continuous response modes. Consider first the dichotomous response mode. Significant practice effects are indicated by the main effects for day $\left[F(1,11)=16.74, M S_{\mathrm{e}}=2.09\right]$ and for block $\left[F(2,22)=11.80, M S_{\mathrm{e}}=1.39\right]$. The interaction of these two variables $\left[F(2,22)=10.95, M S_{\mathrm{e}}=\right.$ 1.22] indicates that the improvement over blocks was smaller on Day 2 than it was on Day 1. That is, learning appears to have reached an asymptote by Day 2 . There also was a main effect of length $[F(2,22)=40.60$, $\left.M S_{\mathrm{e}}=1.68\right]$, indicating a decrease in performance with an increase in sequence length. The modality main effect was not significant $(F<1)$ because of the use of longer sequence lengths for the auditory conditions.

Two higher order interactions also were significant. One interaction was among the modality, day, and block factors $\left[F(2,22)=5.57, M S_{\mathrm{e}}=.61\right]$. This interaction indicates that, on Day 1, the visual sequences showed a greater improvement over blocks than did the auditory sequences. Thus, there is some support for an initial learning spurt for the visual sequences, as predicted by the differential practice hypothesis. The second interaction was among modality, day, and sequence length factors $\left[F(2,22)=3.67, M S_{\mathrm{e}}=.46\right]$. We could find no sensible interpretation for this interaction.

The results from the analyses of the continuous response mode condition were simpler. There were main effects of day $\left[F(1,11)=11.36, M S_{e}=2.69\right]$ and block $\left[F(2,22)=3.45, M S_{\mathrm{e}}=1.10\right]$, both of which indicate practice effects. There also was a main effect of sequence length $\left[F(2,22)=28.28, M S_{\mathrm{e}}=1.23\right]$, indicating a decrease in performance with an increase in sequence length. The main effect of modality was not significant, again because of the use of longer sequence lengths for the auditory conditions. Also, there were no significant interactions involving modality, providing little support to the differential practice hypothesis for this response mode.

We analyzed serial position functions only for the dichotomous response mode, for Day 1, for sequence lengths of 6 and 7 . We do not present the data from the continuous response mode because of the possibility that our scoring procedure (finding a cutoff between long and short responses that maximizes overall probability of being correct) might have had unexpected effects at different serial positions. That is, the cutoff that maximized performance at the early serial positions might have been different from the cutoff that maximized performance at the later serial positions. Furthermore, we do not present the analyses of data from Day 2 because of problems with ceiling effects at many of the serial positions.

The data are presented in Figure 6 . Note that the serial position functions display the same two unusual characteristics that we observed in Experiments 1 and 2, namely, the auditory superiority throughout most of the serial positions and the absence of a strong recency effect.

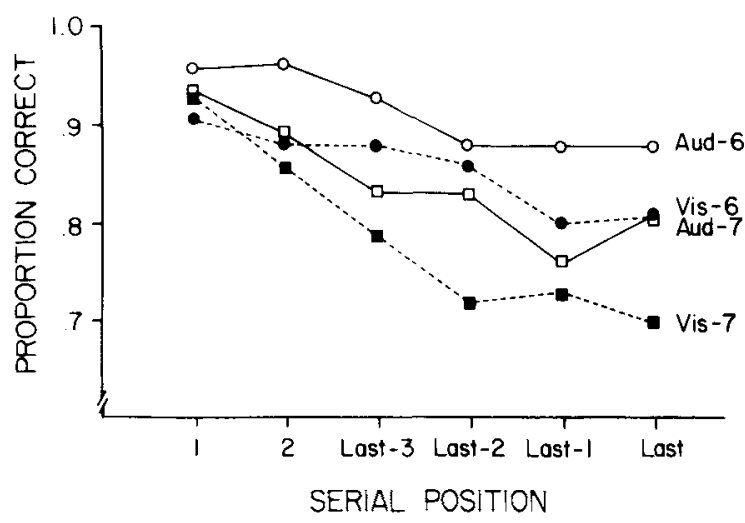

Figure 6. Data from Experiment 3 (dichotomous response mode): Proportion of stimuli correct at each input serial position as a function of modality of presentation and sequence length.

There were main effects of modality $[F(1,11)=9.38$, $\left.M S_{\mathrm{e}}=1.96\right]$, sequence length $\left[F(1,11)=15.16, M S_{\mathrm{e}}=\right.$ 1.76], serial position $\left[F(5,55)=12.61, M S_{\mathrm{e}}=.93\right]$, and block $\left[F(2,22)=12.40, M S_{\mathrm{c}}=1.23\right]$. The main effect of block was due to improvement from .80 to .87 to .89 across Blocks 1, 2, and 3, respectively. Although there is a trend in the data for the auditory superiority to increase across serial positions, the $F$ ratio for the modality by serial position interaction was less than 1.0 .

Three interactions were significant (or nearly so), but they were not of apparent theoretical interest. There was a block $\times$ serial position interaction $[F(10,110)=1.94$, $M S_{\mathrm{e}}=.52 \mathrm{~J}$. Over blocks, there was a $10 \%$ improvement at the last serial position, but only a $3 \%$ improvement at the first serial position, probably because of ceiling effects. The interaction between sequence length and serial position was marginally significant $[F(5,55)=2.29$, $\left.M S_{\mathrm{e}}=.56, p=.058\right]$. As can be seen in Figure 6, the decrease over serial positions was more dramatic for sequences of Length 7 than for sequences of Length 6 . Finally, the block $\times$ sequence length $\times$ serial position interaction was significant $\left[F(10,100)=2.25, M S_{\mathrm{e}}=.33\right]$. The interaction between length and serial position was not apparent in the last block, probably because of ceiling effects. Table 2 provides the means contributing to these interactions.

We draw two new conclusicns from these results. First, because of differences between the modalities in asymptotic performance, we conclude that the auditory superiority does not reflect only differential preexperimental practice with auditory rhythms. There was a hint of differential practice for the dichotomous response mode in that there was an initial learning spurt for the visual sequences that was not matched by the auditory sequences. However, this interaction was not found for the continuous response mode, and, in any case, it did not eliminate the difference between the modalities in asymptotic performance. Second, because the difference between the modalities was also found in the continuous response 
Table 2

Means from Experiment 3:

Dichotomous Response Mode, Day 1, Sequence Lengths 6 and 7

\begin{tabular}{|c|c|c|c|c|c|c|c|}
\hline \multirow[b]{2}{*}{ Block } & \multirow[b]{2}{*}{ Modality } & \multicolumn{6}{|c|}{ Serial Position } \\
\hline & & 1 & 2 & Last 3 & Last 2 & Last 1 & Last \\
\hline \multicolumn{8}{|c|}{ Sequence Length 6} \\
\hline 1 & $\begin{array}{l}\text { Auditory } \\
\text { Visual }\end{array}$ & $\begin{array}{l}.92 \\
.88\end{array}$ & $\begin{array}{l}.95 \\
.78\end{array}$ & $\begin{array}{l}.87 \\
.88\end{array}$ & $\begin{array}{l}.82 \\
.72\end{array}$ & $\begin{array}{l}.82 \\
.65\end{array}$ & $\begin{array}{l}.90 \\
.73\end{array}$ \\
\hline 2 & $\begin{array}{l}\text { Auditory } \\
\text { Visual }\end{array}$ & $\begin{array}{l}.98 \\
.92\end{array}$ & $\begin{array}{l}.98 \\
.93\end{array}$ & $\begin{array}{l}.97 \\
.90\end{array}$ & $\begin{array}{l}.92 \\
.90\end{array}$ & $\begin{array}{l}.88 \\
.85\end{array}$ & $\begin{array}{l}.87 \\
.88\end{array}$ \\
\hline 3 & $\begin{array}{l}\text { Auditory } \\
\text { Visual }\end{array}$ & $\begin{array}{l}.98 \\
.93\end{array}$ & $\begin{array}{l}.98 \\
.92\end{array}$ & $\begin{array}{l}.95 \\
.87\end{array}$ & $\begin{array}{l}.90 \\
.95\end{array}$ & $\begin{array}{l}.95 \\
.90\end{array}$ & $\begin{array}{l}.88 \\
.82\end{array}$ \\
\hline \multicolumn{8}{|c|}{ Sequence Length 7} \\
\hline 1 & $\begin{array}{l}\text { Auditory } \\
\text { Visual }\end{array}$ & $\begin{array}{l}.92 \\
.95\end{array}$ & $\begin{array}{l}.87 \\
.90\end{array}$ & $\begin{array}{l}.73 \\
.75\end{array}$ & $\begin{array}{l}.78 \\
.67\end{array}$ & $\begin{array}{l}.70 \\
.68\end{array}$ & $\begin{array}{l}.75 \\
.60\end{array}$ \\
\hline 2 & $\begin{array}{l}\text { Auditory } \\
\text { Visual }\end{array}$ & $\begin{array}{l}.95 \\
.92\end{array}$ & $\begin{array}{l}.92 \\
.83\end{array}$ & $\begin{array}{l}.88 \\
.78\end{array}$ & $\begin{array}{l}.82 \\
.77\end{array}$ & $\begin{array}{l}.73 \\
.67\end{array}$ & $\begin{array}{l}.82 \\
.70\end{array}$ \\
\hline 3 & $\begin{array}{l}\text { Auditory } \\
\text { Visual }\end{array}$ & $\begin{array}{l}.95 \\
.92\end{array}$ & $\begin{array}{l}.90 \\
.85\end{array}$ & $\begin{array}{l}.88 \\
.85\end{array}$ & $\begin{array}{l}.88 \\
.73\end{array}$ & $\begin{array}{l}.85 \\
.83\end{array}$ & $\begin{array}{l}.87 \\
.80\end{array}$ \\
\hline
\end{tabular}

mode, we conclude that the auditory superiority did not result from a forced incompatible response mode after the visual presentations.

\section{EXPERIMENT 4}

For the sake of parsimony, we would like to maintain that the auditory superiority we have observed here is related (by the temporal coding assumption) to the modality effect observed in recall. Two aspects of the results argue against this relationship, however. First, the modality effect in recall is generally confined to the end of a list, but we observed modality effects throughout the list. It should be noted, however, that through-list modality effects have been reported for recall tasks. Greene (1985), Routh (1976), and J. M. Gardiner, Gregg, and M. M. Gardiner (1984) have reported through-list modality effects in serial recall, and J. M. Gardiner et al. (1984) have found them in free recall.

Second, the modality effect in recall usually takes the form of an enhanced recency effect for auditory material compared to visual material. Here, we observed very little recency effect. However, there are many differences between the rhythm task and standard recall. For one, in the rhythm task, subjects are recalling a sequence of durations; in the standard recall task, we have proposed (Glenberg \& Swanson, 1986) that subjects use temporal information as a retrieval cue to recall other information, namely, the items on the list. This extra stage intervening between the temporal information and recall could well hide any through-list effects (see, for example, Glenberg \& Fernandez, 1988). Another difference between the standard task and the rhythm task is that typically a variety of stimuli is used in the standard task, not just two (long and short) as we used in the rhythm task.

Experiment 4 was designed to determine if the modality effect in recall would resemble the modality effect in the rhythm task when the sequences for the recall task are composed of just two stimuli. In Experiment 4, the subjects experienced four sequence presentation conditions. As in the previous experiments, auditory and visual rhythmic sequences were used. In addition, the subjects recalled sequences composed of the letters $L$ and $S$, which were presented either aurally or visually.

\section{Method}

Subjects. The subjects were 16 members of the University of Wisconsin summer community. Seven were paid for their participation, and the others used participation in Experiment 4 to partially fulfill a course research requirement.

Materials. A new piece of equipment was used to present the rhythmic sequences. This equipment consisted of a small box (resting on top of the video monitor) that enclosed a fixed-frequency squarewave oscillator used to drive a 2 -in. loudspeaker. A diffused lens 5-mm red LED was also part of the box. The Apple I+ computer was used to turn on the oscillator (for auditory rhythmic stimuli) and the LED (for the visual thythmic stimuli) and to time the stimulus duration.

We created the auditory letter stimuli by recording single exemplars of the letters $S$ and $L$ on an Instavox, a device that allows rapid random access to analog recordings of sound. The Instavox was then used to construct a tape recording of the appropriate sequences of the spoken letters, which was amplified and played through the same speaker used to present the auditory rhythmic sequences. The visual letter sequences were presented in the standard Apple Turtlegraphics font on the video monitor controlled by the computer.

All stimulus sequences were 8 elements long. The 12 sequences used in Experiment 4 were constructed from the groups of elements LL, SSL, and LSS (each letter indicates a long or short element for the rhythm task or presentation of the letter $L$ or $S$ in the recall task). Each sequence contained exactly one LL group and two of the other groups (one of each or one used twice). Once three groups were selected (e.g., LL, SSL, LSS), all possible orders of the three groups were constructed, resulting in a set of 12 unique, 8-element sequences. These stimulus sequences were constructed to correspond to those used in another experiment that will not be reported here. Four random orderings of the 12 sequences were generated. These random orderings were assigned in a counterbalanced fashion to the four sequence presentation conditions.

Timing of the rhythmic sequences (including the ready signal and the responses) were as described for Experiments 1-3 (short = $250 \mathrm{msec}$, long $=750 \mathrm{msec}$, interstimulus interval $=250 \mathrm{msec}$ ). For the letter sequences, each letter was presented within a 400-msec window and followed by a $350-\mathrm{msec}$ interval. Thus, the average duration of a letter sequence equaled the average duration of a rhythmic sequence. The ready signal and the response requirements for the letter sequences were exactly the same as those used for the rhythmic sequences.

Design. The two main factors (rhythmic or letter sequence and auditory or visual presentation) were manipulated within subjects, so that each subject experienced all four sequence presentation conditions. The sequences were blocked so that all of the letter sequences appeared first for half of the subjects and all of the rhythmic sequences appeared first for the others. Within these blocks, the sequences were blocked by modality of presentation so that all of the auditory sequences appeared first for half of the subjects and all of the visual sequences appeared first for the others. As noted before, each of the four types of trials was assigned one of the four orders of the 12 unique 8-element sequences. This assignment was counterbalanced across subjects. Each block of 12 trials was 


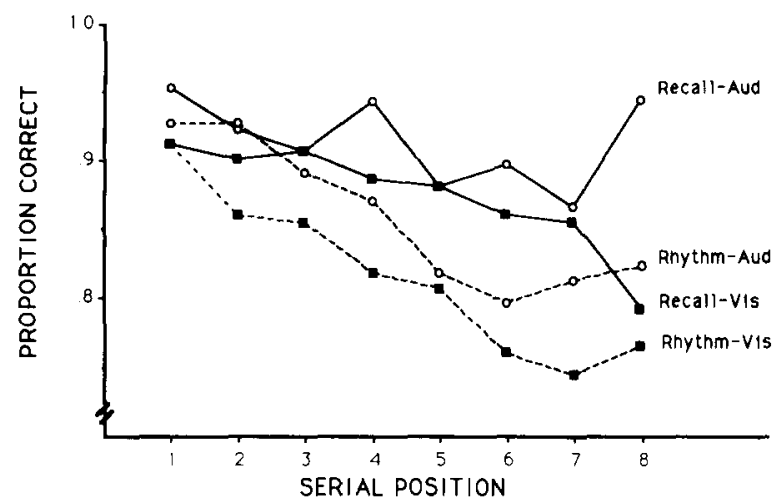

Figure 7. Data from Experiment 4: Proportion of stimuli correct at each input serial position as a function of sequence presentation condition.

preceded by instruction as to the nature of the upcoming trials and two practice trials.

\section{Results and Discussion}

The analysis will focus on the serial position scoring of the 12 sequences in each of the four main conditions. The data are presented in Figure 7.

The main effect for type of sequence $[F(1,15)=7.57$, $\left.M S_{\mathrm{e}}=7.89\right]$ indicates better performance with the letter sequences than with the rhythmic sequences. The main effect of modality $\left[F(1,15)=7.79, M S_{e}=4.04\right]$ indicates better performance with the auditory sequences than with the visual sequences. Finally, there was a main effect of serial position $\left[F(7,105)=11.53, M S_{\mathrm{e}}=2.25\right]$. None of the interactions were significant in this analysis, nor were any interactions significant in an analysis of the last three serial positions.

The major question is whether or not the unusual features of the modality effect in the rhythm task are also found in the recall task, now that we have controlled for the number of stimuli, presentation rate, and so forth. First, judging from Figure 7 and the statistical analyses, the auditory superiority was about the same size in both tasks. Second, for both tasks, the auditory superiority was found in most serial positions, not just at the end of the list. Third, because the interaction between task, modality, and serial position was not significant $(p=.31$, considering all positions; $p=.14$, considering the last three positions), there is not much evidence that the auditory recency effect was larger for the recall task than it was for the rhythm task. However, this last result is weak. Not only is it based on the acceptance of the null hypothesis, but it may well be influenced by a ceiling effect in the recall data. Nonetheless, it is clear from these data that the two modality effects were quite similar.

\section{GENERAL DISCUSSION}

There is a modality effect in the perception and reproduction of rhythms: Auditory rhythmic sequences are reproduced more accurately than are visual rhythmic se- quences. We were able to reject three hypotheses that attributed this auditory superiority to relatively uninteresting processes. The change-from-background hypothesis was ruled out by the results from Experiments 1 and 2: Auditory and visual noise (and intensity of the auditory and visual signals) had little effect on the size of the auditory superiority. The differential learning hypothesis was ruled out by the results of Experiment 3: There was little to suggest that, given enough practice, perception of visual rhythms will approach that of auditory thythms. The results of Experiment 3 also demonstrated that the auditory superiority is not due to specific response requirements.

The remainder of the discussion is organized around three questions. How are the modality effects in the rhythm and recall tasks related? What are the implications of this relationship for explanations of the modality effect? How can we conceptualize this relationship?

There are three reasons for believing that the modality effect found with rhythmic stimuli are produced by the same cognitive mechanisms that produce the modality effect found using letter stimuli. First, the rhythm modality effect is in itself evidence that the processes that produce the standard modality effect are being tapped. Of course, one could propose that modality of presentation influences multiple information processing stages and that different stages are responsible for the two modality effects. Nonetheless, until evidence for this claim is made available, it is more parsimonious to assume that the two modality effects are produced by the same underlying processes.

On the basis of the results of Experiments 1-3, one could argue that the rhythm modality effect is different from the standard modality effect, because it is found at all serial positions and because it is not associated with a large recency effect. However, this argument is at least partially nullified by the results of Experiment 4: The unusual features of the rhythm modality effect were also characteristic of the recall modality effect when the number of stimuli was reduced to two. Thus, the fact that the shapes of the serial position functions in the rhythm and recall tasks were similar is a second reason for believing that the two modality effects tap the same mechanisms.

Glenberg, Jona, and Curran (1989) discuss a third reason. Their reasoning is based on a correlational analysis: If two effects are produced by the same information processing mechanism, the sizes of the effects should correlate across subjects. In fact, Glenberg et al. found a correlation of .61 between the size of the modality effect in a rhythm task and the size of the modality effect in a more standard recall task. For reasons discussed in Glenberg et al.'s manuscript, application of this analysis to the data from Experiment 4 is open to a number of artifacts, and thus will not be reported. ${ }^{3}$

If, indeed, the rhythm and recall modality effects are produced by the same cognitive processes, what are the implications for explanations of the modality effect? First, we note that our results using the rhythm task bear a family 
resemblance to Roberts's (1986) finding of modality effects in memory for melodic stimuli (see also Greene \& Samuel, 1986). Roberts presented to trained musicians sequences of musical notes or the visual counterparts of the notes using musical notation. As in Experiments 1-4, Roberts found auditory superiority that was not confined to the recency portion of the list, and she found relatively small recency effects. Thus, Roberts's results (as pointed out by Greene \& Samuel, 1986), as well as the results from Experiments 1-4, demonstrate that auditory superiority is not necessarily linked to speech. Therefore, speech-based explanations of the modality effect are, at best, incomplete. Two such explanations are the Crowder and Morton (1969) speech-based precategorical acoustic store model and the Shand and Klima (1981) primary linguistic code hypothesis. The latter hypothesis is that the modality effect results from coding information using the code most often used by the perceiver for coding linguistic information.

We can make an even stronger claim. To the extent that the rhythm modality effect and the recall modality effect tap the same mechanisms (and Experiment 4 and Glenberg et al.'s 1989 data suggest that they do), we can discount speech-based hypotheses as viable accounts of any modality effect. That is, these hypotheses cannot account for the rhythm modality effect, and because the rhythm and recall modality effects are (probably) produced by the same mechanisms, the hypotheses cannot adequately explain the recall modality effect.

The results from Experiment 1 (in which background was manipulated) are also inconsistent with Nairne's (1988) framework for interpreting recency effects. Nairne proposed that visual recency effects are modest because visual features of the ambient visual environment interfere with the recently encoded visually presented items. This account would predict that, compared to the nobackground condition, the visual-noise-background condition would severely degrade performance on the visual sequences. The data in Figure 1 provide little comfort for this position.

The rhythm modality effect also has positive implications in that it supports the idea that modality effects reflect the coding of temporal information. That is, the rhythm modality effect is consistent with the claim (J. M. Gardiner, 1983; Glenberg \& Swanson, 1986) that auditory stimuli result in more accurate coding of temporal information than do visual stimuli. Rhythms are, of course, temporal stimuli, and the very fact that the rhythm modality effect is obtained with a pure temporal stimulus is suggestive that temporal mechanisms are contributing to the effect.

Glenberg et al. (1989) report data that are, perhaps, more convincing that the rhythm modality effect (and thus, by implication, the recall modality effect) reflects the coding of temporal information. Glenberg et al. disrupted the rhythmic (temporal) quality of the stimulus sequences by randomly varying the duration of the interstimulus interval between the elements in the rhythm task. This manipu- lation eliminated the rhythm modality effect by reducing performance on the auditory sequences to that on the visual sequences, whereas the manipulation had little effect on performance on the visual sequences. Thus, this purely temporal manipulation affects auditory sequences but not visual sequences-a result that strongly supports the assumption that temporal information plays a more important role in memory for auditory events than in memory for visual events.

We have yet to describe, however, exactly how temporal coding is affected by modality. One hypothesis, derived from the reports of our subjects, attributes modality differences to the coding of duration. Many subjects claimed that they attempted to code the visual stimuli by quietly humming. Perhaps then, duration is encoded by a single mechanism that is stimulated directly by auditory signals, but requires intervention (e.g., humming) to encode duration of visual signals. For example, Baddeley and Salamé (1986) phonetic store could be one such mechanism. Variability in beginning or ending the intervention could make discriminating long and short visual stimuli more difficult than discriminating long and short auditory stimuli. Alternatively, the intervention may not activate the time encoder to the same degree as direct stimulation.

A second hypothesis concerns the coding of sequential, or ordinal, relations among stimuli. This hypothesis is based on research investigating the coding of time of presentation of events (rather than of duration). This research indicates that the representation of time of occurrence is unlikely to be akin to a tag that gives clock time. Instead, it appears that time of presentation is coded as an ordinal relation, that the target event occurred after some other event, or simultaneously with another (Tzeng \& Cotton, 1980; Winograd \& Soloway, 1985). In addition, there is some evidence that this sort of relational coding is hierarchical, both in the short term (Lee \& Estes, 1981) and the long term (Friedman, 1987; Friedman \& Wilkins, 1985).

Following Winograd and Soloway (1985), suppose that when one event reminds the learner of a previous event, an ordinal (temporal) relation is coded between the two events. Suppose further that this reminding can be based on perceptual similarities as well as conceptual similarities. Now, why might this form of ordinal coding be superior for auditory events than for visual events? There are many possibilities, but one that appeals to us is based on the notion of auditory streaming (Bregman, 1981; Bregman \& Campbell, 1971). An auditory stream is a type of perceptual organization imposed on the auditory environment. The stream is the equivalent of an auditory object. Whether or not successive auditory events stream depends on similarity of spatial location, pitch, and rhythm (Handel, Weaver, \& Lawson, 1983). Thus, perhaps the auditory events used in Experiments 1-4 stream, enhancing the perception of similarity. Because of this perceived similarity, ordinal relations are coded between the representations of successive tones (or auditory letters) more 
readily than they are between the representations of successive asterisks (or visual letters). The result is a finegrained coding of order relations for auditory events but not for visual events, much as proposed by the temporal coding assumption.

\section{REFERENCES}

Atkinson, R. C., \& Shiffrin, R. M. (1968). Human memory: A proposed system and its control processes. In K. W. Spence \& J. T. Spence (Eds.), The psychology of learning and motivation (Vol. 2, pp. 89-105). New York: Academic Press.

Baddeley, A., \& Salamé, P. (1986). The unattended speech effect: Perception or memory. Journal of Experimental Psychology: Learning, Memory, \& Cognition, 12, 525-529.

BREGMAN, A. S. (1981). Asking the "what for" question in auditory perception. In M. Kubovy \& J. R. Pomerantz (Eds.), Perceptual organization (pp. 99-118). Hillsdale, NJ: Erlbaum.

Bregman, A. S., \& Campbell, J. (1971). Primary auditory stream segregation and perception of order in rapid sequences. Journal of Experimental Psychology, 89, 244-249.

Crowder, R. G., \& GreEne, R. L. (1987). On the remembrance of times past: The irregular list technique. Journal of Experimental Psychology: General, 116, 265-278.

Crowder, R. G., Morton, J. (1969). Precategorical acoustic storage (PAS). Perception \& Psychophysics, 5, 365-377.

FrIedman, W. J. (1987). A follow-up to "Scale Effects in Memory for the Time of Events": The earthquake study. Memory \& Cognition, 15, 518-520.

Friedman, W. J., \& Wilkins, A. (1985). Scale effects in memory of the time of events. Memory \& Cognition, 13, 168-175.

Gardiner, J. M. (1983). On recency and echoic memory. Philosophical Transactions of the Royal Society of London, 302, 267-282.

GARDINER, J. M., \& GREGG, V. H. (1979). When auditory memory is not overwritten. Joumal of Verbal Learning \& Verbal Behavior, 18 , 705-719.

Gardiner, J. M., GregG, V. H., \& Gardiner, M. M. (1984). Concerning some more evidence of an auditory advantage in prerecency as well as recency recall. American Journal of Psychology, 97, 593-604.

Glenberg, A. M., \& Fernandez, A. (1988). Evidence for auditory temporal distinctiveness: Modality effects in order and frequency judgments. Journal of Experimental Psychology: Learming, Memory, \& Cognition, 14, 728-739.

Glenkerg, A. M., \& Jona, M., \& CURRAN, T. (1989). Temporal coding in rhythm and recall tasks revealed by modality effects. Unpublished manuscript.

Glenberg, A. M., Swanson, N. C. (1986). A temporal distinctiveness theory of recency and modality effects. Journal of Experimental Psychology: Learning, Memory, \& Cognition, 12, 3-15.

GreEne, R. L. (1985). Constraints on the long-term modality effect. Joumal of Memory \& Language, 24, 526-541.
Greene, R. L., \& Samuel, A. G. (1986). Recency and suffix effects in serial recall of musical stimuli. Journal of Experimental Psychology: Learning, Memory, \& Cognition, 12, 517-524.

Handel, S., Weaver, M. S., Lawson, G. (1983). Effect of thythmic grouping on stream segregation. Journal of Experimental Psychology: Human Perception \& Performance, 9, 637-651.

LEE, C. L., \& EsTES, W. K. (1981). Item and order information in shortterm memory: Evidence for multilevel perturbation process. Journal of Experimental Psychology: Human Leaming \& Memory, 7, 149-169.

Metcalfe, J., Glavanov, D., \& Murdock, M. (1981). Spatial and temporal processing in the auditory and visual modalities. Memory \& Cognition, 9, 351-359.

NAIRNE, J. S. (1988). A framework for interpreting recency effects in immediate and serial recall. Memory \& Cognition, 16, 343-352.

Posner, M. I., Nissen, M. J., Klein, R. M. (1976). Visual dominance: An information-processing account of its origins and significance. Psychological Review, 83, 157-171.

Roberts, L. A. (1986). Modality and suffix effects in memory for melodic and harmonic musical materials. Cognitive Psychology, 18, 123-157.

Routh, D. A. (1976). An "across-the-board" modality effect in immediate serial recall. Quarterly Joumal of Experimental Psychology, 28, 285-304.

Shand, M. A., \& Kuma, E. S. (1981). Nonauditory suffix effects in congenitally deaf signers of American Sign Language. Joumal of Experimental Psychology: Human Learning \& Memory, 7, 464-474.

TzENG, O. J. L., Corton, B. (1980). A study-phase retrieval model of temporal coding. Journal of Experimental Psychology: Human Learming \& Memory, 6, 705-716.

Winograd, E., Soloway, R. M. (1985). Reminding as a basis for temporal judgments. Joumal of Experimental Psychology: Leaming, Memory, \& Cognition, 11, 262-271.

\section{NOTES}

1. These experiments were designed in part to check on the following thought experiment. Imagine a drummer on television. Is it easier to perceive the rhythm while listening with your eyes closed, or is it easier to perceive the thythm while watching with the sound off? Our intuition was that the former would be easier.

2 . The tone was produced by a software procedure that created a series of rapid clicks that were amplified by an external amplifier. The call to the tone procedure was embedded within the routines that created the visual background noise and the asterisks. When there was no need for a tone, volume of the tone was set to zero. Similarly, when there was no need for the noise background, its intensity was set to zero. In this way, the timing and other characteristics of the tones and asterisks were identical across conditions.

3. In brief, an appropriate correlational analysis must equate the four presentation conditions for variability, level of performance, and reliability. See Glenberg et al. (1989) for details.

(Manuscript received January 15, 1988; revision accepted for publication October 31,1988 .) 